\title{
Multiple retrograde substructures in the Galactic halo: A shattered view of Galactic history
}

\author{
Helmer H. Koppelman ${ }^{1}$, Amina Helmi ${ }^{1}$, Davide Massari ${ }^{1,2,3}$, Adrian M. Price-Whelan ${ }^{4}$, and Tjitske K. Starkenburg ${ }^{4}$
}

\author{
1 Kapteyn Astronomical Institute, University of Groningen, Landleven 12, 9747 AD Groningen, The Netherlands \\ e-mail: koppelman@astro.rug.nl \\ 2 Dipartimento di Fisica e Astronomia, Università degli Studi di Bologna, Via Gobetti 93/2, 40129 Bologna, Italy \\ 3 INAF - Osservatorio di Astrofisica e Scienza dello Spazio di Bologna, Via Gobetti 93/3, 40129 Bologna, Italy \\ 4 Center for Computational Astrophysics, Flatiron Institute, 162 5th Avenue, New York, NY 10010, USA
}

Received 19 September 2019 / Accepted 24 October 2019

\begin{abstract}
Aims. Several kinematic and chemical substructures have been recently found amongst Milky Way halo stars with retrograde motions. It is currently unclear how these various structures are related to each other. This Letter aims to shed light on this issue. Methods. We explore the retrograde halo with an augmented version of the Gaia DR2 RVS sample, extended with data from three large spectroscopic surveys, namely RAVE, APOGEE, and LAMOST. In this dataset, we identify several structures using the HDBSCAN clustering algorithm. We discuss their properties and possible links using all the available chemical and dynamical information.

Results. In concordance with previous work, we find that stars with $[\mathrm{Fe} / \mathrm{H}]<-1$ have more retrograde motions than those with $[\mathrm{Fe} / \mathrm{H}]>-1$. The retrograde halo contains a mixture of debris from objects like Gaia-Enceladus, Sequoia, and even the chemically defined thick disc. We find that the Sequoia has a smaller range in orbital energies than previously suggested and is confined to high energy. Sequoia could be a small galaxy in itself, but since it overlaps both in integrals-of-motion space and chemical abundance space with the less bound debris of Gaia-Enceladus, its nature cannot yet be fully settled. In the low-energy part of the halo, we find evidence for at least one more distinct structure: Thamnos. Stars in Thamnos are on low-inclination, mildly eccentric retrograde orbits, moving at $v_{\phi} \approx-150 \mathrm{~km} \mathrm{~s}^{-1}$, and are chemically distinct from the other structures.

Conclusions. Even with the excellent Gaia DR2 data, piecing together all the fragments found in the retrograde halo remains challenging. At this point, we are very much in need of large datasets with high-quality high-resolution spectra and tailored high-resolution hydrodynamical simulations of galaxy mergers.
\end{abstract}

Key words. Galaxy: halo - solar neighborhood - Galaxy: kinematics and dynamics - Galaxy: formation - Galaxy: evolution

\section{Introduction}

A wide variety of cosmological simulations, typically performed in a $\Lambda$ cold dark matter $(\Lambda \mathrm{CDM})$ setting, have shown that the stellar halo of the Milky Way is an excellent testbed for galaxy formation models (Helmi et al. 2003; Bullock \& Johnston 2005; Johnston et al. 2008; Cooper et al. 2010; Pillepich et al. 2014; Grand et al. 2017). In $\Lambda$ CDM, the halos of galaxies like the Milky Way grow in size by merging with other galaxies, mostly through minor mergers. Galaxies that merge leave behind debris in the form of a trail of stars, and at the solar position this debris typically is very phase-mixed (Helmi \& White 1999). Disentangling the superimposed trails of different mergers is in principle possible with the help of detailed dynamical information like the integrals of motion (Helmi \& de Zeeuw 2000), or the actions (McMillan \& Binney 2008). In a local volume, each stream (a portion of a trail with stars with similar orbital phase) has typically a very low density, and has been estimated to contain on average $0.25 \%$ and at maximum $5 \%$ of the total number of local halo stars (Gould 2003).

The outer stellar halo of the Milky Way is consistent with being completely built up through mergers (e.g. Belokurov et al. 2006; Bell et al. 2008; Helmi et al. 2011). With Gaia (Gaia Collaboration 2016, 2018) it has become possible to map the kinematics of the local stellar halo in great detail (e.g. Helmi et al. 2017; Myeong et al. 2018a,b; Koppelman et al. 2018). An impressive finding in the field of Galactic archaeology since the release of Gaia DR2 is the debris of Gaia-Enceladus-Sausage (Belokurov et al. 2018; Helmi et al. 2018): a massive dwarf galaxy that contributed a large fraction of the local stellar halo. The initial stellar mass of this object was $5 \times 10^{8}-5 \times 10^{9} M_{\odot}$ (Belokurov et al. 2018; Helmi et al. 2018; Mackereth et al. 2019; Vincenzo et al. 2019) and it was accreted $\sim 10 \mathrm{Gyr}$ ago (Helmi et al. 2018; Di Matteo et al. 2018; Gallart et al. 2019). We refer to this dwarf as Gaia-Enceladus, but the kinematic footprint of this dwarf galaxy is sometimes also referred to as Gaia-Sausage.

Besides Gaia-Enceladus, the Helmi streams (Helmi et al. 1999) are located in the prograde part of the halo. These streams originate in a dwarf galaxy of $M_{\star} \sim 10^{8} M_{\odot}$ that was accreted 5-8 Gyr ago (Koppelman et al. 2019, see also Kepley et al. 2007). While a large fraction of stars with retrograde motions appears to be debris from Gaia-Enceladus, especially for high-eccentricity (Helmi et al. 2018, see also Belokurov et al. 2018), for very retrograde motions $\left(v_{\phi}<-100 \mathrm{~km} \mathrm{~s}^{-1}\right)$ the situation is less clear. This portion of the halo contains several small structures (e.g. Myeong et al. 2018b; Koppelman et al. 2018; Matsuno et al. 2019), and plausibly also debris of Gaia-Enceladus. Also, Mackereth et al. (2019) postulate that the low-eccentricity region had a more complex formation history and would be composed by a mixture of stars formed in situ, debris from Gaia-Enceladus, and debris 
from other structures. One such structure would be the Sequoia (Myeong et al. 2019), whose existence builds on the discovery of a large globular cluster with very retrograde halo-like motion, FSR-1758 (Barba et al. 2019).

In this Letter we quantify the degree of clustering in a local sample of halo stars using both dynamical and metallicity information. This allows us to discover debris from another small object, which we term Thamnos, as well as to establish on firmer grounds the reality and relationship between the different structures reported thus far in the literature in this rapidly evolving field.

\section{Data}

We use here an augmented version of the Gaia RVS sample, extended with radial velocities from APOGEE DR14 (Abolfathi et al. 2018), LAMOST (Cui et al. 2012), and RAVE DR5 (Kunder et al. 2017); see Sects. 2.1 and 2.2 of Koppelman et al. (2019) for more details. Because the metallicity scales of the three different surveys are not necessarily the same, we use the LAMOST values, unless stated otherwise. The results do not depend on this choice, except that the cross-matches with APOGEE and RAVE have considerably fewer stars. In total, our sample comprises 8738322 stars with full 6D phase-space information and high-quality parallaxes (parallax_over error $>5$ ) of which 3404432 have additional [Fe/H] information and 189444 have chemical abundances from APOGEE. To calculate the distance we invert the parallaxes. Even when using high-quality parallaxes, biases in the distances could be introduced by inverting the parallaxes. However, we find that the structures identified in this work are robust to using other distance estimates such as those provided by McMillan (2018) or Schönrich et al. (2019). Because of the systematic parallax offset in Gaia DR2 (Arenou et al. 2018; Gaia Collaboration 2018; Lindegren et al. 2018), which for the RVS sample might even be more significant (Schönrich et al. 2019), we restrict our analysis to stars within $3 \mathrm{kpc}$ of the Sun. When inspecting velocities we use a selection of stars in an even smaller volume to optimise the amount of clumpiness (by avoiding possible velocity gradients).

The velocities of the stars are corrected for the solar motion assuming $(U, V, W)=(11.1,12.24,7.25) \mathrm{km} \mathrm{s}^{-1}$ (Schönrich et al. 2010), and for the motion of the local standard of rest (LSR) using $v_{\mathrm{LSR}}=232.8 \mathrm{~km} \mathrm{~s}^{-1}$ (McMillan 2017). Cartesian coordinates are calculated such that $X$ points towards the Galactic Centre, and $Y$ points in the direction of the motion of the disc. Cylindrical coordinates are derived in a right-handed system, although we flip the sign of $v_{\phi}$ such that it coincides with the $Y$-axis at the solar position. In this system, the Sun is located at $X=-8.2 \mathrm{kpc}$. We use the implementation of the McMillan (2017) potential in AGAMA (Vasiliev 2019) to calculate orbital parameters such as the total energy (En), eccentricity (ecc), circularity ( $c i r c)$, apocentre (apo), and pericentre (peri). The circularity is calculated as circ $=$ $L_{z} /\left|L_{z \text {,circ }}\right|$, where $L_{z, \text { circ }}$ is the vertical component of the angular momentum for a circular orbit with the same $E n$ of the star.

In this work, we identify halo stars by their kinematics, a selection mostly used for illustrative purposes. As we are mainly interested in the retrograde halo we impose a relatively conservative cut by removing stars with $\left|\boldsymbol{V}-\boldsymbol{V}_{\mathrm{LSR}}\right|<230 \mathrm{~km} \mathrm{~s}^{-1}$.

\section{Results}

\subsection{The metal-poor, retrograde halo}

Figure 1 shows a velocity diagram of the local stellar halo (distance $<1 \mathrm{kpc}$ ) split in a metal-poor (top) and a metal-rich

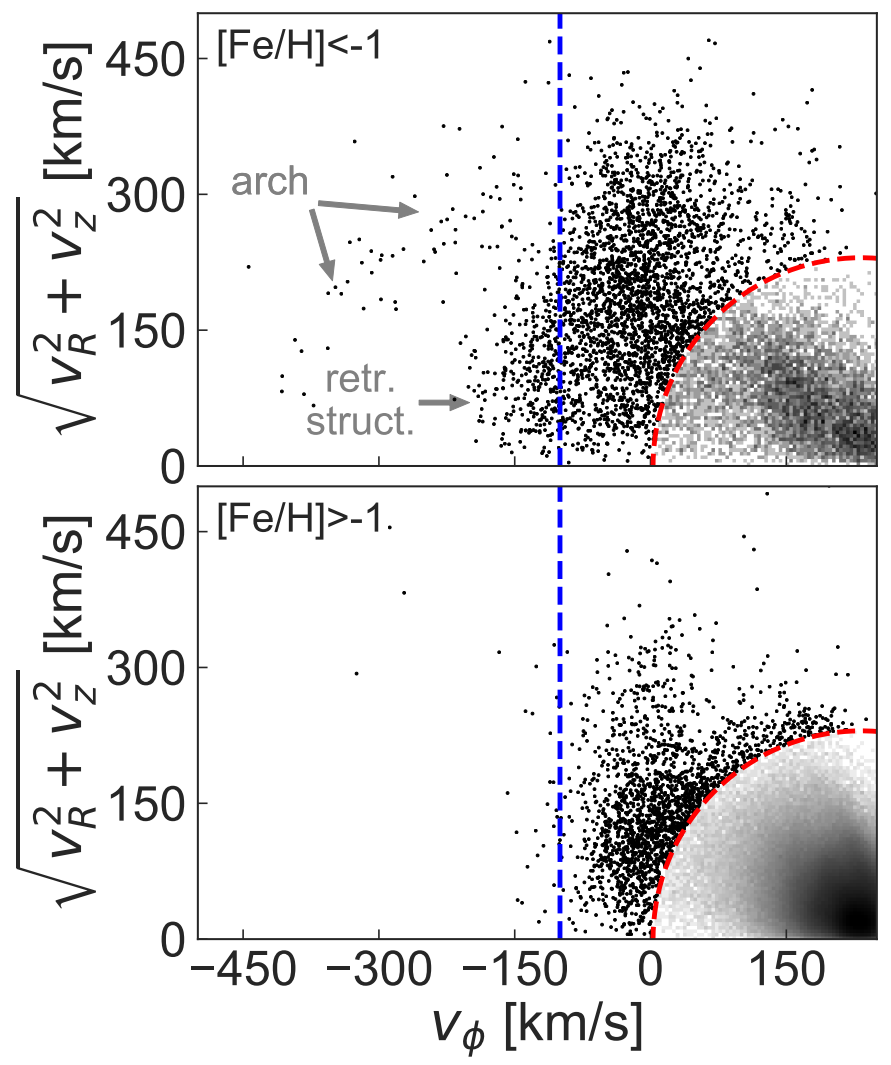

Fig. 1. Velocity diagram of the local $(d<1 \mathrm{kpc})$ stellar halo split in a metal-poor (top) and a metal-rich sample (bottom). The colour-coding of the 2D histogram scales with the logarithm of the number of stars in each bin. All stars outside of the red dashed line are tentatively labelled as halo stars and are shown as black dots. We note that most if not all of the halo left of the dashed vertical line $\left(v_{\phi}<-100 \mathrm{~km} \mathrm{~s}^{-1}\right)$ is more metal-poor than $[\mathrm{Fe} / \mathrm{H}]=-1$.

(bottom) sample. Two-dimensional histograms show the distribution of all the stars in the given $[\mathrm{Fe} / \mathrm{H}]$ selection, while halo stars are highlighted with small black dots. The vertical dashed line indicates the very retrograde limit and highlights the large amount of small-scale substructure present for low metallicity. This is consistent with previous work reporting that the retrograde halo is more metal-poor (e.g. Carollo et al. 2007; Matsuno et al. 2019; Myeong et al. 2019). One of the structures seen is the arch reaching from $\left(v_{\phi},\left(v_{z}^{2}+v_{R}^{2}\right)^{1 / 2}\right)=(-100,300)$ to $(-450,0) \mathrm{km} \mathrm{s}^{-1}$, which overlaps with the retrograde structures of Myeong et al. (2018b) and with the red and purple structures in Koppelman et al. (2018). The arch was associated to GaiaEnceladus (Helmi et al. 2018) on the basis of resemblance to the simulations of Villalobos \& Helmi (2008).

Besides the arch, there is another retrograde structure apparent in the metal-poor halo, at $v_{\phi}=-150 \mathrm{~km} \mathrm{~s}^{-1}$ and with $\left(v_{z}^{2}+\right.$ $\left.v_{R}^{2}\right)^{1 / 2}<150 \mathrm{~km} \mathrm{~s}^{-1}$, that is with counter-rotating thick-disc-like kinematics. A subset of this retrograde component was picked up as the VelHel-4 structure Helmi et al. (2017) and as the blue and orange structures reported in Koppelman et al. (2018).

The debris of Gaia-Enceladus, which we identify here as the dominant contributor to the halo in the range $-100<v_{\phi}<$ $50 \mathrm{~km} \mathrm{~s}^{-1}$, has more stars with $[\mathrm{Fe} / \mathrm{H}]<-1$ (top panel) but also contributes to the metal-rich (bottom) panel. The only structure that is more abundant in the metal-rich part of the halo is the extension of the thick-disc, identified as the slow-rotating tail of the thick-disc (e.g. Koppelman et al. 2018; Haywood et al. 2018; 
Di Matteo et al. 2018). Stars with thin-disc-like motions appear to also exist with $[\mathrm{Fe} / \mathrm{H}]<-1$.

\subsection{Selecting distinct substructures}

Figure 1 on its own does not clear up if and how the retrograde structures are related. To study this in more detail we apply the clustering algorithm $\operatorname{HDBSCAN}^{1}(\mathrm{McInnes}$ et al. 2017). We use the default parameters of the algorithm, after setting min_samples $=3$, min_cluster_size $=15$, and cluster_selection_method = "leaf". These settings, especially the leaf mode, tune the algorithm to find fine-grained structure instead of large overdensities. In our experience, no clustering algorithm is capable of picking out each of the halo overdensities given the large amount of overlap, the measurement errors, and the lack of metallicities for most sources. Therefore, we aim to break up the halo into small, robust groups that can be used to trace the large structures. Based on these groups we then place selection boxes to select the larger structures.

As input parameters for the algorithm we use $E n, L_{z}, e c c$, and $[\mathrm{Fe} / \mathrm{H}]$, which are all often used to find substructure in the stellar halo (e.g. Helmi \& de Zeeuw 2000; Helmi et al. 2017; Koppelman et al. 2018; Mackereth et al. 2019). The space that is defined by these parameters is scaled with RobustScaler implemented in scikit-learn (Pedregosa et al. 2011) using the default settings of the code. We select all stars within $3 \mathrm{kpc}$ of the Sun and $\left|\boldsymbol{V}-\boldsymbol{V}_{\mathrm{LSR}}\right|>180 \mathrm{~km} \mathrm{~s}^{-1}$, because we are mainly interested in picking up structure in the halo. This selection includes a significant amount of thick-disc stars that should be identified as a distinct component if the algorithm works properly. There are no thin-disc stars in this selection.

Figure 2 shows the stars associated with substructures according to HDBSCAN, colour-coded by $[\mathrm{Fe} / \mathrm{H}]$, while the remaining stars are shown with black dots. The top-left panel is similar to Fig. 1 and shows a very clear gradient of metallicity with $v_{\phi}$. Both the arch and the low $\left(v_{z}^{2}+v_{R}^{2}\right)^{1 / 2}$ structures are picked up as (metal-poor) groups (in yellow), while the thickdisc is also apparent (in purple). When varying the HDBSCAN parameters the individual groups change slightly, but the large structures which they trace persist. The results are also unaffected by changes in the limiting distance of the stars, at least up to $5 \mathrm{kpc}$ from the Sun.

The top-right panel of Fig. 2 shows the distribution of the clusters in $E n-L_{z}$ space. In this space, it becomes clear that the arch structure strongly overlaps with the retrograde group (i.e. Sequoia) identified by Myeong et al. (2018b). As a reference we add the globular clusters FSR 1758 and $\omega$-Cen to this diagram, both of which have tentatively been assigned to the Sequoia by Myeong et al. (2019, although Massari et al. 2019 argues that the latter is more likely associated with Gaia-Enceladus). In the bottom-right panel of Fig. 2 we have overlaid lines of constant circularity (circ $=-0.2,-0.4,-0.6$ ), with solid lines corresponding to circular orbits in the Galactic plane. We select here regions occupied predominantly by the various structures as follows:

- Gaia-Enceladus: $-1.5<E n /\left[10^{5} \mathrm{~km}^{2} \mathrm{~s}^{-2}\right]<-1.1$ and $-0.20<\operatorname{circ}<0.13$;

- Sequoia: $-1.35<E n /\left[10^{5} \mathrm{~km}^{2} \mathrm{~s}^{-2}\right]<-1.0$ and $-0.65<$ circ $<-0.4$

\footnotetext{
1 Hierarchical Density-Based Spatial Clustering of Applications with Noise, a clustering algorithm that excels over the better known DBSCAN both because it is less sensitive to the parameter selection and because it can find clusters of varying densities. See also https://hdbscan . readthedocs.io
}

- The low $\left(v_{z}^{2}+v_{R}^{2}\right)^{1 / 2}$ structure is split in two based on the different metallicities: (i): with $v_{\phi} \sim-200 \mathrm{~km} \mathrm{~s}^{-1}$, circ $<-0.75$ and $-1.65<E n /\left[10^{5} \mathrm{~km}^{2} \mathrm{~s}^{-2}\right]<-1.45$; and (ii): with $v_{\phi} \sim-150 \mathrm{~km} \mathrm{~s}^{-1},-0.75<\operatorname{circ}<-0.4$ and $-1.8<$ $\mathrm{En} /\left[10^{5} \mathrm{~km}^{2} \mathrm{~s}^{-2}\right]<-1.6$.

We use different colours to show how the stars in these selections are distributed in velocity space in the bottom-left panel of Fig. 2. As a reference, we also plot the Helmi streams (HStr), selected as all stars with $1600<L_{\perp} /\left[\mathrm{kpc} \mathrm{km} \mathrm{s}^{-1}\right]<3200$ and $1000<L_{z} /\left[\mathrm{kpc} \mathrm{km} \mathrm{s}^{-1}\right]<1500$ (cf. Koppelman et al. 2019). We note that in this figure, no globular clusters are found on the region occupied by the stars colour-coded dark blue (cf. Massari et al. 2019). On the other hand, the cyan stars are located near $\omega$-Cen and therefore if we follow the argument of Myeong et al. (2018b), they could belong to the Sequoia. It is possible however that these cyan stars are tracing a new structure, are associated with those in the dark blue selection, or are a mixture of several structures.

For completeness, in Fig. 3 we plot the structures in other projections of velocity space. Their distribution is highly reminiscent of the simulated substructures in Helmi \& de Zeeuw (2000), suggesting that they could indeed belong to different dwarf-galaxy progenitors.

\subsection{Chemical analysis}

Figure 4 shows the distribution of stars in our sample with abundances from APOGEE (with ASPCAPFLAG $==0$ ), colour coded according to our selections. Particularly for $[\mathrm{Fe} / \mathrm{H}]>-1.5$, we see that the stars in cyan reveal contamination from the chemically defined thick disc as indicated by the annotation (despite their very retrograde motion) and Gaia-Enceladus. For low $[\mathrm{Fe} / \mathrm{H}]$, these stars typically have higher $[\mathrm{Mg} / \mathrm{Fe}]$ than Sequoia and Gaia-Enceladus, indicating a different origin. We tentatively refer to the structure defined by the cyan and dark blue stars as Thamnos, that is "shrubs", because these stars stand at the foot of a Greek giant and a tall tree in both velocity and $E n-L_{z}$ spaces. We keep for now the distinction between the stars with $v_{\phi} \sim-200 \mathrm{~km} \mathrm{~s}^{-1}$ and those with $v_{\phi} \sim-150 \mathrm{~km} \mathrm{~s}^{-1}$ and refer to them as Thamnos 1 and 2, respectively.

The Sequoia stars on the other hand overlap with the metalpoor tail of Gaia-Enceladus, making it difficult to argue that they truly originate in a different system. It should be noted that much of this analysis is tentative as it is only based on a small sample of stars and at low $[\mathrm{Fe} / \mathrm{H}]$ the errors are significant. Furthermore, the various other (independent) elements in APOGEE also have errors that are too large to be of help. With the amounts of data coming in the next few years this analysis will be much improved.

\section{Discussion and conclusions}

We used Gaia DR2 data supplemented with line-of-sight velocities and chemical abundances from RAVE, APOGEE, and LAMOST to shed more light on the nearby stellar halo and its substructures. The stars in the retrograde halo are predominantly metal-poor. In fact, as the motions of the stars become more retrograde, the stars are even more metal-poor (i.e. a "gradient" in $v_{\phi}$ with $\left.[\mathrm{Fe} / \mathrm{H}]\right)$. This gradient is reminiscent of the dual halo reported in Carollo et al. (2007), but its nature is more complex. Our analysis seems to suggest that the outer halo is more retrograde because it is dominated by debris from (the outskirts of) Gaia-Enceladus and Sequoia. This was already hinted at by 

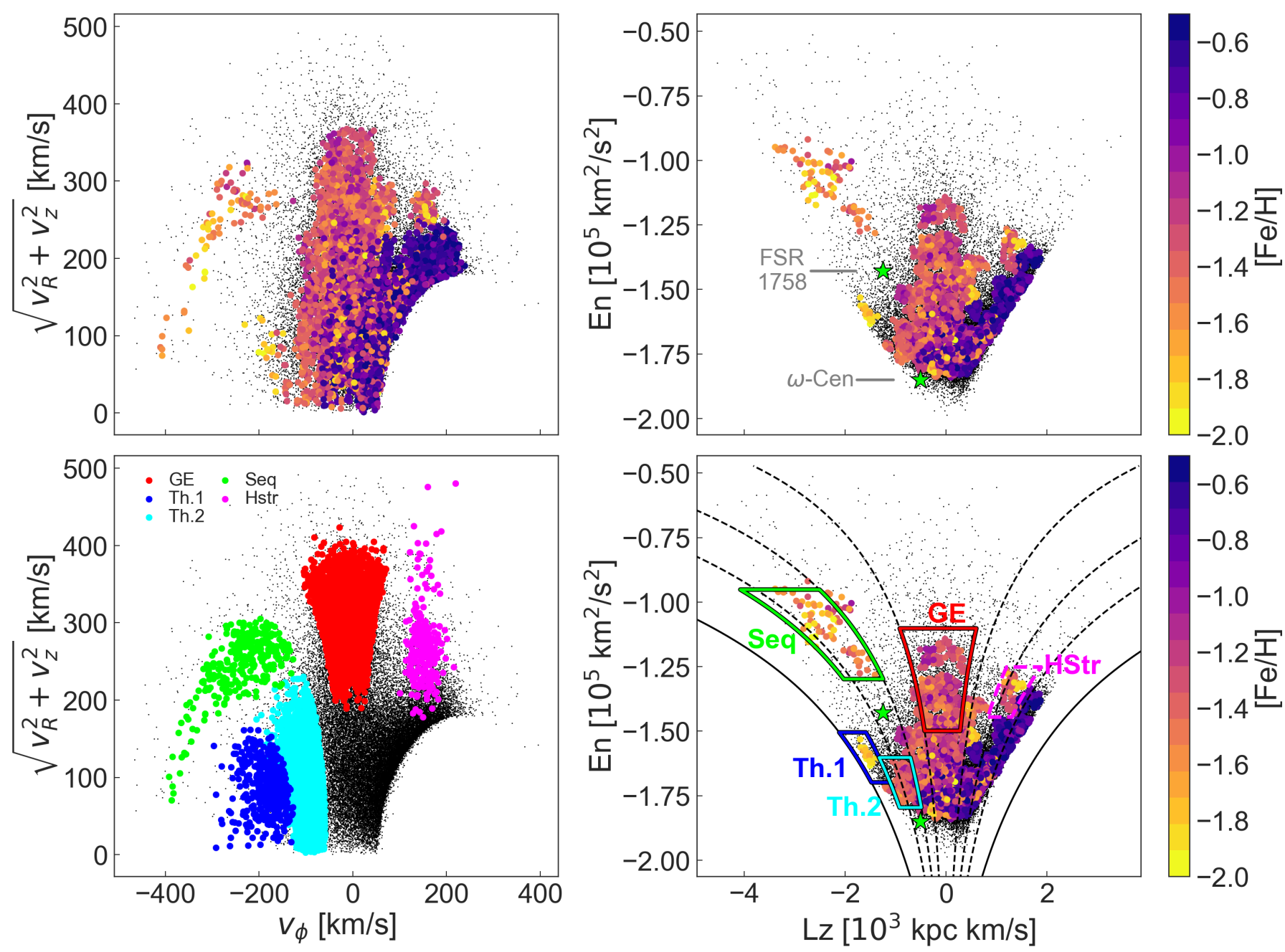

Fig. 2. Top row: distribution of the stars in the groups identified by HDBSCAN in $E n, L_{z}$, ecc, and $[\mathrm{Fe} / \mathrm{H}]$ space, and colour-coded by [Fe/H], with the rest shown with black dots. Bottom-right panel: over-plotted lines of constant circularity and used coloured boxes to indicate our selection of substructures. We note that the Helmi Streams (HStr) are selected in $L_{z}-L_{\perp}$ space as described in the text. Bottom-left panel: kinematic properties of the stars in these substructures.

Helmi et al. (2017), who have shown that at high energies the halo is retrograde. On the other hand, stars on very retrograde motions with orbits in the inner halo belong to a newly identified (but previously reported in part in Helmi et al. 2017; Koppelman et al. 2018; Mackereth et al. 2019) substructure which we have named Thamnos.

\subsection{Notes on Sequoia}

Using a mixture of spectroscopic data, the Stellar Abundances for Galactic Archeology (SAGA) database (Suda et al. 2008), Matsuno et al. (2019) have shown that the trend defined by Sequoia members is slightly offset from that of Gaia-Enceladus. Despite the fact that we only have three stars with full abundance information provided by APOGEE, we derive a similar conclusion.

In spite of using the same APOGEE dataset as Myeong et al. (2019) we reach different conclusions on the nature of Sequoia. One of the reasons driving this is that we find that it is more natural to separate the very retrograde halo into a high- $E n$ (Sequoia) and a low-En (Thamnos) substructures. Sequoia is not only chemically different from Thamnos, but it seems also difficult to reconcile its metallicity with the large extent in En pro- posed by Myeong et al. (2019, starting from the more unbound Gaia-Enceladus debris down to the energy of $\omega$-Cen). Such a large range in energy can only be produced by a very massive object, as illustrated in Fig. 5 where we have overlaid contours on the $E n-L_{z}$ diagram using the extent of mock dwarf galaxies. The outer contour corresponds to a mock dwarf galaxy of $M_{\star}=10^{8} M_{\odot}$ and the inner to $M_{\star}=5 \times 10^{6} M_{\odot}$ (see for details on the mock dwarfs Sect. 5 of Koppelman et al. 2019). The mocks are centred on an orbit that is chosen to be roughly in the centre of the debris of Sequoia and Thamnos in this diagram (only slight shifts are found when a different central orbit is chosen). The contours encompass $80 \%$ of the stars in the mock dwarfs. Therefore the extent of an object in $E n-L_{z}$ space reflects - to some degree - the initial mass and size of the progenitor. Figure 5 shows that the contours for the Sequoia and Thamnos do not overlap, confirming our assessment that these are likely distinct systems.

On the other hand, having lower binding energy and more retrograde motion than the bulk of the debris of Gaia-Enceladus and overlapping with its metal-poor tail, Sequoia could well be, at least in part, debris from the outer regions of Gaia-Enceladus (see Fig. 1 of Helmi et al. 2018), lost at early times. This analysis suggests that at best we are dealing with a bonsai Sequoia. 

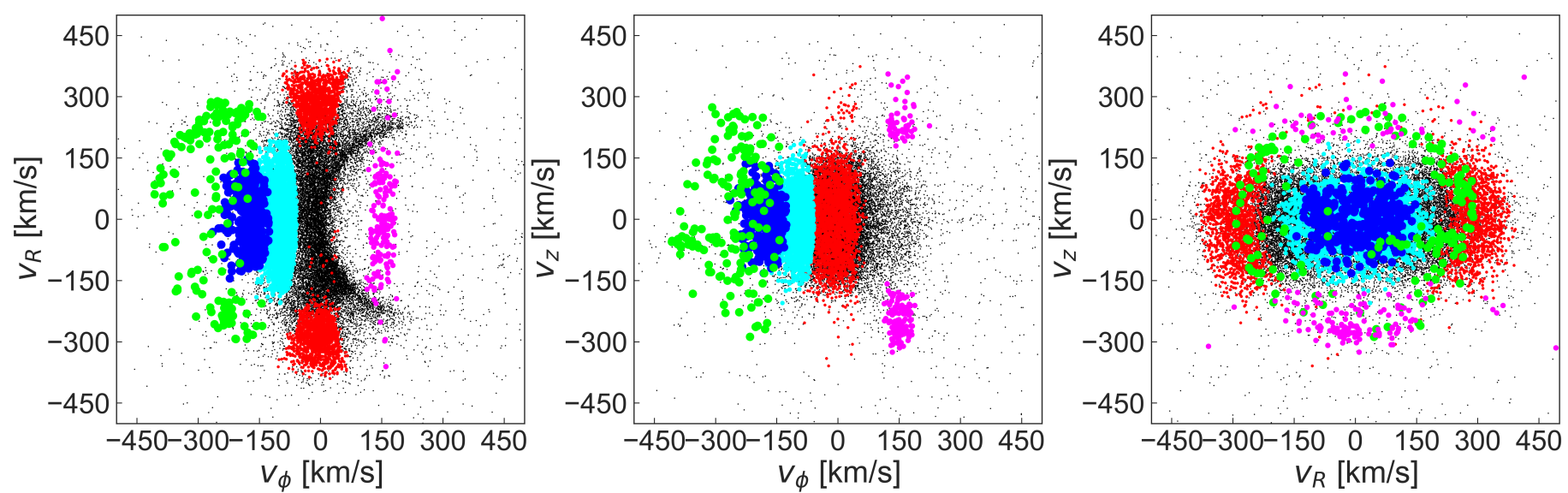

Fig. 3. Velocity distributions of the stars in the structures identified in Fig. 2 (using the same colour-coding), with non-selected stars with halo-like kinematics stars in black. Only stars within $2 \mathrm{kpc}$ are shown here to optimise the amount of clumpiness in this space (by minimising velocity gradients).
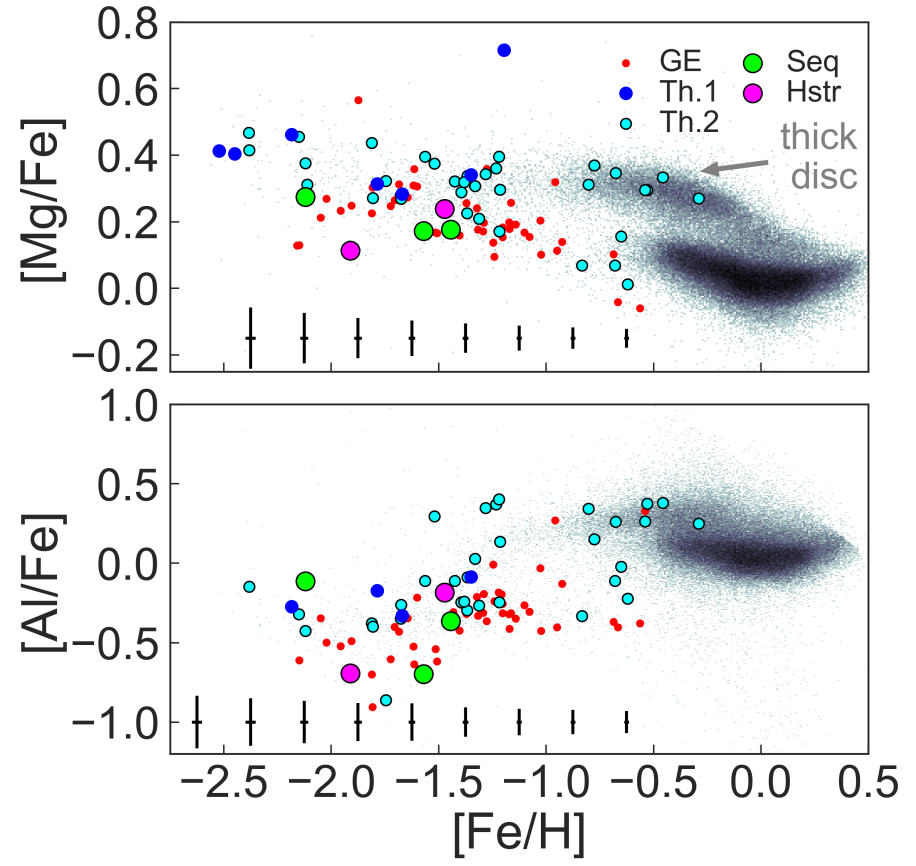

Fig. 4. Detailed chemical abundances (from APOGEE) for stars in the different substructures. The bars at the bottom of the panel indicate the mean error at that $[\mathrm{Fe} / \mathrm{H}]$. In the background we show a $2 \mathrm{D}$ histogram of all of the stars in our dataset, colour-coded by the logarithm of the number of stars per bin.

\subsection{Thamnos}

We find evidence for one or two more distinct components in the local retrograde halo: Thamnos 1 and 2. The debris of these objects is characterised by strong retrograde rotation and high binding energy. Especially the values of En suggests that these structures may have been accreted a very long time ago. The distribution of these structures in $E n-L_{z}$ space is compatible with them originating in the same dwarf galaxy; see Fig. 5. Figure 2 (bottom, right) shows that neither $\omega$-Cen nor FSR 1758 fall inside the selection boxes for Thamnos. When comparing to the full catalogue of Massari et al. (2019), we find no globular clusters to fall inside the selection for Thamnos. Compared to GaiaEnceladus, the chemical composition of the stars of Thamnos are more metal-poor and significantly more $\alpha$-enhanced. As far as

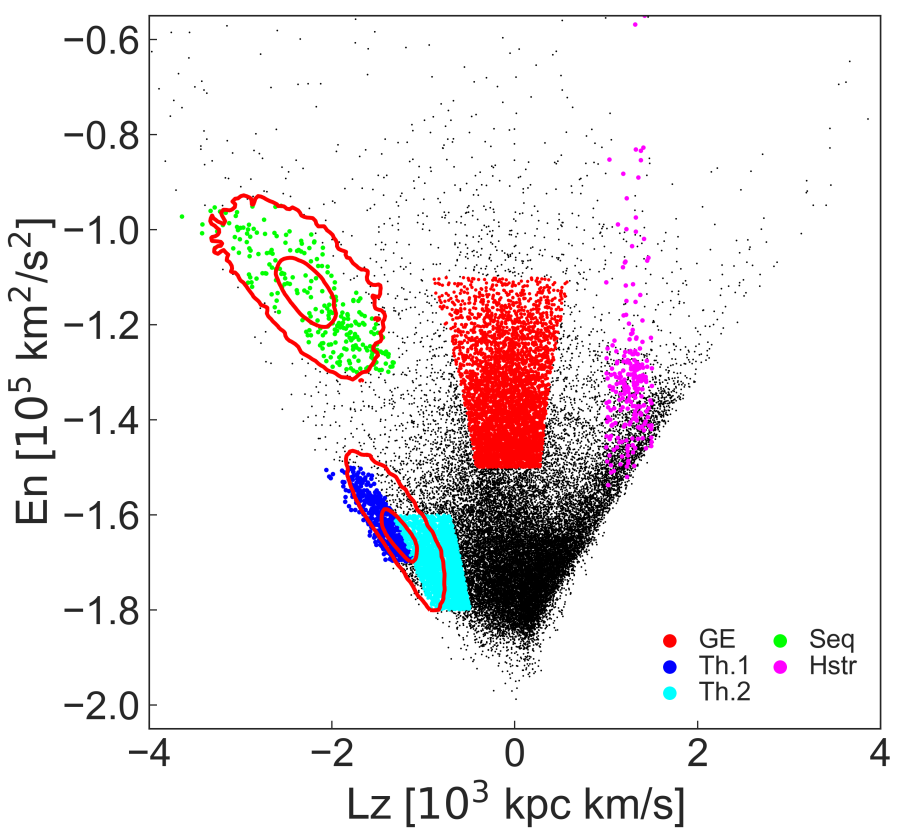

Fig. 5. Distribution of stars in $E n-L_{z}$ space, where the contours indicate the extent of mock dwarf galaxies placed on top of the debris of Sequoia and Thamnos 1 and 2 . The inner contour is for a dwarf galaxy of $M_{\star}=$ $5 \times 10^{6} M_{\odot}$ and the outer for $M_{\star}=10^{8} M_{\odot}$. In the background we show all stars with halo-like kinematics within $3 \mathrm{kpc}$ with small dots, see Sect. 2, with those belonging to selected structures colour-coded as in Fig. 2.

we can judge and given their similar abundances, Thamnos 1 and 2 share the same progenitor whose stellar mass $M_{\star} \lesssim 5 \times 10^{6} M_{\odot}$.

\subsection{The chemically defined thick-disc}

Our analysis reveals the presence of stars from the thick-disc with retrograde motions, identified chemically because they are metal-rich and $\alpha$-enhanced. It will be interesting to study these stars detailed chemical composition: they are amongst the oldest stars that formed in the in situ disc of the Milky Way. Since they were present at the time of the merging of GaiaEnceladus (which explains their hot orbits) such a study would allow the characterisation of the disc at $z \gtrsim 2$. Early attempts of such studies have dated the merger event of Gaia-Enceladus 
(Di Matteo et al. 2018) and found an ultra metal-poor disc component (Sestito et al. 2019).

\subsection{Final note}

The main conclusion of this work is that even with the excellent Gaia DR2 data, putting the shattered pieces together to reconstruct history, as in true Galactic archaeology, remains challenging at present. The different substructures identified in dynamical space show significant overlap. Chemical tagging helps with disentangling, but the current sample of high-quality and reliable abundances is too small to lead to firm conclusions. At this point we are in desperate need for high-quality spectroscopic observations of the halo stars to supplement the Gaia data, as fortunately planned for WEAVE (Dalton et al. 2012) and 4MOST (de Jong et al. 2012). Furthermore, significant progress could be made by comparing the detailed properties of the substructures to tailored high-resolution hydrodynamical simulations of mergers of satellites with Milky Way-like galaxies.

Acknowledgements. $\mathrm{HHK}$ and $\mathrm{AH}$ acknowledge financial support from a Vici grant from NWO. The Flatiron Institute is supported by the Simons Foundation. This work has made use of data from the European Space Agency (ESA) mission Gaia (http://www. cosmos.esa.int/gaia), processed by the Gaia Data Processing and Analysis Consortium (DPAC, http://www . cosmos. esa int/web/gaia/dpac/consortium). Funding for the DPAC has been provided by national institutions, in particular the institutions participating in the Gaia Multilateral Agreement. In the analysis, the following software packages have been used: vaex (Breddels \& Veljanoski 2018), numpy (Van Der Walt 2011), matplotlib (Hunter 2007), jupyter notebooks (Kluyver et al. 2016).

\section{References}

Abolfathi, B., Aguado, D. S., Aguilar, G., et al. 2018, ApJS, 235, 42 Arenou, F., Luri, X., Babusiaux, C., et al. 2018, A\&A, 616, A29

Barba, R. H., Minniti, D., Geisler, D., et al. 2019, ApJ, 870, L24

Bell, E. F., Zucker, D. B., Belokurov, V., et al. 2008, ApJ, 680, 295

Belokurov, V., Zucker, D. B., Evans, N. W., et al. 2006, ApJ, 642, L137

Belokurov, V., Erkal, D., Evans, N. W., Koposov, S. E., \& Deason, A. J. 2018 MNRAS, 478, 611

Breddels, M. A., \& Veljanoski, J. 2018, A\&A, 618, A13

Bullock, J. S., \& Johnston, K. V. 2005, ApJ, 635, 931

Carollo, D., Beers, T. C., Lee, Y. S., et al. 2007, Nature, 450, 1020

Cooper, A. P., Cole, S., Frenk, C. S., et al. 2010, MNRAS, 406, 744

Cui, X.-Q., Zhao, Y.-H., Chu, Y.-Q., et al. 2012, Res. Astron. Astrophys., 12, 1197

Dalton, G., Trager, S. C., Abrams, D. C., et al. 2012, in Ground-based and Airborne Instrumentation for Astronomy IV, eds. I. S. McLean, S. K. Ramsay, \& H. Takami, Int. Soc. Opt. Photon., 8446, 84460P de Jong, R. S., Bellido-Tirado, O., Chiappini, C., et al. 2012, in Ground-based and Airborne Instrumentation for Astronomy IV, eds. I. S. McLean, S. K. Ramsay, \& H. Takami, Int. Soc. Opt. Photon., 8446, 84460T

Di Matteo, P., Haywood, M., Lehnert, M. D., et al. 2018, A\&A, 604, A106

Gaia Collaboration (Prusti, T., et al.) 2016, A\&A, 595, A1

Gaia Collaboration (Brown, A. G. A., et al.) 2018, A\&A, 616, A21

Gallart, C., Bernard, E. J., Brook, C. B., et al. 2019, Nat. Astron., 3, 932

Gould, A. 2003, ApJ, 592, 63

Grand, R. J. J., Gómez, F. A., Marinacci, F., et al. 2017, MNRAS, 467, 179

Haywood, M., Di Matteo, P., Lehnert, M. D., et al. 2018, ApJ, 863, 113

Helmi, A., \& de Zeeuw, P. T. 2000, MNRAS, 319, 657

Helmi, A., \& White, S. D. M. 1999, MNRAS, 307, 495

Helmi, A., White, S. D. M., De Zeeuw, P. T., et al. 1999, Nature, 402, 53

Helmi, A., White, S. D. M., \& Springel, V. 2003, MNRAS, 339, 834

Helmi, A., Cooper, A. P., White, S. D. M., et al. 2011, ApJ, 733, L7

Helmi, A., Veljanoski, J., Breddels, M. A., Tian, H., \& Sales, L. V. 2017, A\&A, 598, A58

Helmi, A., Babusiaux, C., Koppelman, H. H., et al. 2018, Nature, 563, 85

Hunter, J. D. 2007, Comput. Sci. Eng., 9, 90

Johnston, K. V., Bullock, J. S., Sharma, S., et al. 2008, ApJ, 689, 936

Kepley, A. A., Morrison, H. L., Helmi, A., et al. 2007, ApJ, 134, 1579

Kluyver, T., Ragan-Kelley, B., Pérez, F., et al. 2016, Jupyter Notebooks-a Publishing Format for Reproducible Computational Workflows (IOS Press)

Koppelman, H. H., Helmi, A., \& Veljanoski, J. 2018, ApJ, 860, L11

Koppelman, H. H., Helmi, A., Massari, D., Roelenga, S., \& Bastian, U. 2019, A\&A, 625, A5

Kunder, A., Kordopatis, G., Steinmetz, M., et al. 2017, AJ, 1, 12

Lindegren, L., Hernandez, J., Bombrun, A., et al. 2018, A\&A, 616, A2

Mackereth, J. T., Schiavon, R. P., Pfeffer, J., et al. 2019, MNRAS, 482, 3426

Massari, D., Koppelman, H. H., \& Helmi, A. 2019, A\&A, 630, L4

Matsuno, T., Aoki, W., \& Suda, T. 2019, ApJ, 874, L35

McInnes, L., Healy, J., \& Astels, S. 2017, J. Open Sour. Softw., 2, 2

McMillan, P. J. 2017, MNRAS, 465, 76

McMillan, P. J. 2018, Res. Notes Am. Astron. Soc., 2, 51

McMillan, P. J., \& Binney, J. J. 2008, MNRAS, 390, 429

Myeong, G. C., Evans, N. W., Belokurov, V., Amorisco, N. C., \& Koposov, S. E. 2018a, MNRAS, 475, 1537

Myeong, G. C., Evans, N. W., Belokurov, V., Sanders, J. L., \& Koposov, S. E. 2018b, MNRAS, 478, 5449

Myeong, G. C., Vasiliev, E., Iorio, G., Evans, N. W., \& Belokurov, V. 2019, MNRAS, 488, 1235

Pedregosa, F., Varoquaux, G., Gramfort, A., et al. 2011, J. Mach. Learn. Res., 12,2825

Pillepich, A., Vogelsberger, M., Deason, A., et al. 2014, MNRAS, 444, 237

Schönrich, R., Binney, J., \& Dehnen, W. 2010, MNRAS, 403, 1829

Schönrich, R., Mcmillan, P., \& Eyer, L. 2019, MNRAS, 487, 3568

Sestito, F., Longeard, N., Martin, N. F., et al. 2019, MNRAS, 484, 2166

Suda, T., Katsuta, Y., Yamada, S., et al. 2008, PASJ, 60, 1159

Van Der Walt, S. 2011, The NumPy Array: A Structure for Efficient Numerical Computation (IEEE)

Vasiliev, E. 2019, MNRAS, 482, 1525

Villalobos, A., \& Helmi, A. 2008, MNRAS, 391, 1806

Vincenzo, F., Spitoni, E., Calura, F., et al. 2019, MNRAS, 487, 47 